\title{
L'obésité : d'une adaptation sociétale à une maladie organique du tissu adipeux
}

Bernard GUY-GRAND

Président du GLN,

Professeur honoraire de nutrition

En introduction aux conférences qui vont suivre lors de cette septième journée du Groupe Lipides et Nutrition consacrée aux relations entre lipides alimentaires et obésité, il est sans doute utile de rappeler quelques éléments importants, issus des réflexions du groupe de I'Hôtel-Dieu menées depuis des années en commun avec Arnaud Basdevant, Michelle Le Barzic puis Jean-Michel Oppert et Karine Clément [1] : la prise de poids puis le surpoids et l'obésité sont en grande partie la conséquence des adaptations aux transformations sociétales du monde dit «moderne»; en elles-mêmes elles ne sont pas dans la plupart des cas « maladives » mais aboutissent à modifier de façon durable et en grande partie irréversible le tissu adipeux, véritable support organique d'un état qui devient pathologique.

Surpoids et obésité sont des situations chroniques sans tendance spontanée à la régression une fois qu'elles sont constituées et cliniquement très hétérogènes. Cette hétérogénéité entre les sujets obèses (aussi manifeste que celle existant entre les sujets normaux) est telle qu'il conviendrait toujours de parler « des obésités ». Autre caractéristique: ces états de surpoids-obésité sont d'origine multifactorielle : il n'y a pas une façon unique de devenir obèse, ce qui complique beaucoup l'analyse du phénomène où l'on se doit de combiner une grande hétérogénéité clinique et une vaste multiplicité de facteurs.

\section{Une définition en partie arbitraire}

Inflation du tissu adipeux, l'obésité est définie, par convention, par l'indice de masse corporelle (IMC) dont les valeurs permettent de distinguer différents degrés (tableau 1) en fonction des risques statistiques de morbi-mortalité qui lui sont statistiquement affectés. Même si elle est commode et universellement acceptée, cette définition souffre de plusieurs faiblesses qui la rendent en partie arbitraire et approximative.

L'IMC reflète correctement l'importance de la masse grasse (coefficient de l'ordre de 0,8) au sein d'une population mais n'en est qu'un indicateur grossier pour un individu donné : pour un même IMC le pourcentage de masse grasse peut varier du simple au double.

Le risque de morbi-mortalité des sujets obèses n'est pas lié qu'à l'IMC mais dépend aussi de la distribution du tissu adipeux dans I'organisme : le risque cardiométabolique, notamment, est fortement lié au développement du tissu adipeux intra-abdominal que l'IMC n'évalue pas. La mesure du tour de taille cherche à caractériser ces « obésités abdominales » avec plus ou moins d'approximations.

Les relations entre IMC et risque cardiométabolique varient d'une population à l'autre : pour les Asiatiques par exemple, le niveau de risque pour un IMC de 25 est de l'ordre de grandeur de celui d'un «Caucasien » (Européen) avec un IMC de 30 .

La substitution de I'IMC au pourcentage de masse grasse n'est donc qu'une approximation commode puisque celui-ci reste difficile à mesurer en pratique même si la méthode de l'impédancemétrie fait des progrès. (Pour la recherche, seule l'absorptiométrie biphotonique (DEXA), voire, avec des réserves, la pléthysmographie, doivent être utilisées.)
Il faut donc se garder de caractériser les sujets uniquement sur la foi de leur IMC sans prendre en compte une vaste série d'autres éléments.

\section{Épidémiologie}

Forte prévalence et rapide expansion depuis quelques décennies (1 à 4) selon les pays, prennent I'allure d'une pandémie mondiale caractérisent l'obésité.

En France, comme l'indique le tableau 2, I'étude OBEPI, réalisée par la Sofres tous les 3 ans depuis 1997 sous la direction de l'Inserm à l'initiative des laboratoires Roche documente cette évolution.

On y constate qu'en 9 ans l'obésité (IMC > 30) a progressé de $56 \%$ pour atteindre au moins $12,4 \%$ de la population adulte, l'obésité «morbide » de $166 \%$. II est trop tôt pour savoir si le léger fléchissement observé pour le surpoids relève d'une tendance stable liée aux politiques de santé ou d'un simple effet de cohorte, puisque l'étude n'est pas longitudinale mais transversale.

Cette étude montre que le phénomène touche toutes les tranches d'âge et décèle un effet générationnel : la prévalence de l'obésité chez les sujets nés entre 1973 et 1979 à 30 ans est la même que celle des sujets de 40 ans... 10 ans plus tôt.

En fait, les chiffres du tableau 2 sont sans doute sous-évalués puisque l'IMC y est calculé à partir

Tableau 1. Les différents degrés de l'indice de masse corporelle (IMC), fonction des risques statistiques de morbimortalité qui lui sont statistiquement affectés. Source : OMS-IOTF-IASO.

\begin{tabular}{|lcc|}
\hline & IMC $\mathbf{( k g} / \mathbf{m}^{\mathbf{2}} \mathbf{)}$ & Risque \\
\hline Sous-poids & $<18,5$ & Élevé \\
Zone normale & $18,5-25$ & Moyen \\
Surpoids - Préobèse & $25-29,9$ & Augmenté \\
Obésité classe I & $30-34,9$ & Modéré \\
Obésité classe II & $35-39,9$ & Sévère \\
Obésité classe III (morbide) & $\geq 40$ & Très sévère \\
\hline
\end{tabular}


Tableau 2. Prévalence du surpoids et de l'obésité en France dans la population adulte (étude OBEPI : Institut Roche de l'Obésité, Inserm, Sofres).

\begin{tabular}{|lcccccc|}
\hline IMC & & $\mathbf{1 9 9 7}$ & $\mathbf{2 0 0 0}$ & $\mathbf{2 0 0 3}$ & $\mathbf{2 0 0 6}$ & $\mathbf{1 9 9 7 / 2 0 0 6}$ \\
\hline $25-29,9$ & Spds & $28,5 \%$ & $29,4 \%$ & $30,3 \%$ & $29,2 \%$ & $+2,4 \%$ \\
$30-34,9$ & $I$ & 6,8 & 7,7 & 8,9 & 9,4 & $+51 \%$ \\
$35-39,9$ & II & 1,1 & 1,5 & 1,8 & 2,2 & \\
$>40$ & III & 0,3 & 0,4 & 0,6 & 0,8 & \\
& & $8,2 \%$ & $9,6 \%$ & $11,3 \%$ & $12,4 \%$ & $+166 \%$ \\
\hline
\end{tabular}

des poids (souvent sous-estimés) et des tailles (souvent surestimées) rapportés par les sujets. La réalité est sans doute supérieure de 3 à 4 points.

Signalons enfin que la prévalence de l'obésité culmine dans les classes sociales les moins favorisées et les moins acculturées.

Chez l'enfant, pour lequel l'obésité est plus difficile à définir, la prévalence de l'obésité et du surpoids est sans doute encore plus forte, avoisinant 16 à $18 \%$ entre 5 et 12 ans, ce qui laisse prévoir que dans les décennies qui viennent les chiffres chez l'adulte augmenteront environ $70 \%$ des enfants et adolescents obèses le restent à l'âge adulte.

L'obésité est un phénomène mondial, qui frappe intensément les États-Unis, où la prévalence est passée entre 1990 et 2005 de $10 \%$ à plus de $25 \%$ dans beaucoup d'États, mais aussi les pays dits émergents comme la Chine et I'Inde où la progression, en milieu urbain, est nettement plus rapide. En Chine, quand un sujet du Céleste Empire achète une voiture et remise son vélo au garage, il prend en moyenne $3,5 \mathrm{~kg}$ dans l'année : on n'insistera jamais assez sur l'importance de l'activité physique, au moins autant que sur celle du Coca et de Mac Do auxquels ces populations sont confrontées depuis peu de temps.

\section{Obésité : une maladie par ses conséquences}

II fait peu de doute que les conséquences médicales de l'obésité soient assez considérables pour que l'OMS ait souhaité qu'elle soit considérée comme une maladie.

Diabète de type 2, hypertension artérielle, maladie coronarienne, lithiase biliaire entre autres sont fortement déterminées par la valeur de I'IMC comme le montre la figure 1. Cette influence apparaît pour des valeurs d'IMC considérées comme de «simples» surpoids. Des IMC compris entre 28 et 30 à l'âge moyen de la vie (45-54 ans) sont associés à une augmentation de $50 \%$ de la mortalité. Surpoids et obésité figurent ainsi parmi les premières causes de mortalité prématurée (évitable ?). Par extrapolation d'estimations américaines on peut sans doute considérer que 30 à 45000 décès par an sont dus au poids excessif, ce qui lot). Ces mécanismes régulateurs sont nettement plus efficaces pour lutter contre les déficits que pour compenser les excès qui ne présentent pas de conséquences vitales à court terme, ce qui représentait même un avantage dans un passé assez proche où accumuler des réserves protégeait contre les périodes de famine.

L'efficacité de la régulation ainsi que le niveau auquel se situeront les réserves énergétiques varient beaucoup en fonction des caractéristiques génétiques propres à chacun et de l'environnement plus ou moins favorisant dans lequel l'individu se trouve placé ; ces deux catégories de facteurs exercent leurs effets en interaction permanente.

En dehors de rares cas d'obésités monogéniques d'expression phénotypique plus ou moins obligatoire, les variants géniques favorisant l'obésité sont des "gènes de susceptibilité ", agissant en interaction les uns avec les autres et conditionnant un certain degré de vulnérabilité vis-à-vis de l'obésité. Un très grand nombre de régions du génome sont ainsi impliquées de façon plus ou moins précise sans que I'on connaisse exactement la nature des gènes impliqués. Ils peuvent agir à différents niveaux, aussi bien celui des dépenses énergétiques (par exemple, les sujets qui ont un métabolisme de repos en dessous de la moyenne ont beaucoup plus de risques que ceux qui sont au-dessus de la moyenne), que des différents éléments qui contrôlent la prise alimentaire ou l'efficacité des réponses neurohormonales aux changements d'environnement.

Les facteurs d'environnement qualifiés « d'obésitogènes» sont multiples. On peut constater que les modifications relativement récentes de l'environnement et des modes de vie relèvent de cette qualification et sont les éléments moteurs de l'épidémie d'obésité, d'où l'idée que l'obésité relève dans beaucoup bilan d'énergie (un même phénomène existe, l'échelle cellulaire, cf. l'exposé de Michel Bey-

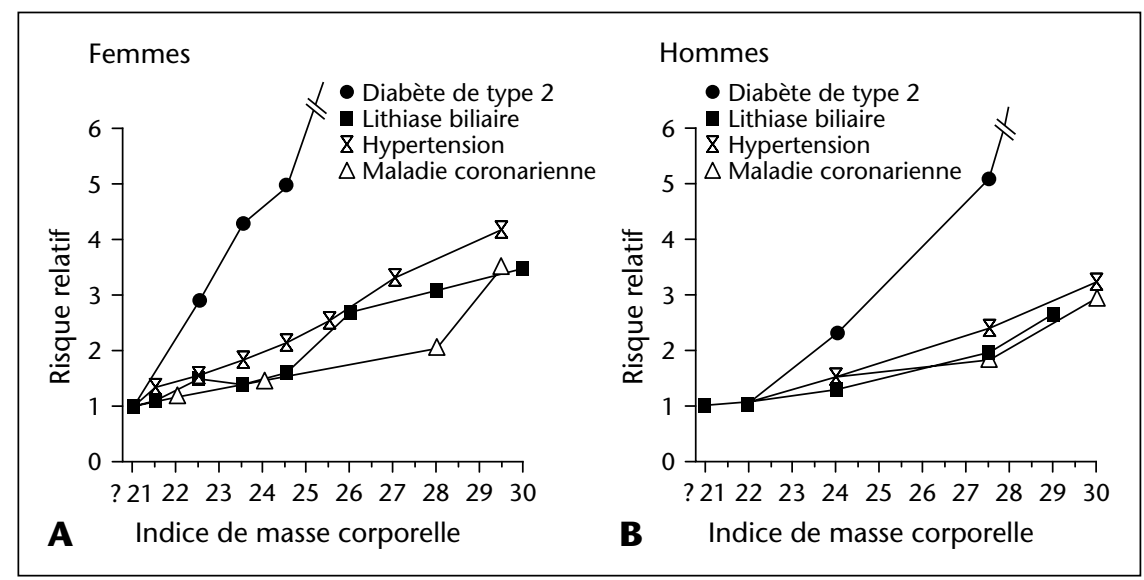

Figure 1. Corrélation entre la valeur de l'IMC et le risque relatif de développer un diabète de type 2, de l'hypertension

artérielle, une maladie coronarienne, et une lithiase biliaire. D'après From Willett et al. NEJM 1999.

en ferait la deuxième cause de mortalité prématurée après le tabagisme. publique, au coût social et économique considérable (coûts directs évalués à 2 à $3 \%$ des c'est aussi un problème individuel auquel doi-

Une discussion approfondie n'a pas sa place La prise de poids (de graisses de réserve) relève d'un mécanisme fondamental unique : I'excès ses (bian d'énergie par rapport aux qu'il y a plus de 50 manières de provoquer La relative constance de l'état des réserves energétiques est assurée par des mécanismes dent à faire varier en sens contraire entrées alimentaires et dépenses énergétiques de façon à compenser les écarts en plus ou en moins $d u$ 
de cas d'une adaptation à un écosystème changeant. Ils agissent non seulement en tant que tels pour diminuer les dépenses énergétiques et pousser à l'excès alimentaire, mais aussi en modifiant le fonctionnement des gènes à travers les mécanismes épigénétiques de modification de l'expression génique au cours du développement (prénatal, petite enfance...). Les modifications du milieu intérieur secondaires à la constitution de l'obésité (hyperinsulinémie, hypercortisolisme...) rétroagissant sur la plupart des mécanismes biologiques (expression génique en particulier) tout comme l'état d'obésité lui-même modifie les comportements du sujet.

Ainsi se constituent une série de cercles vicieux qui rendent compte de l'autoentretien de l'obésité et de son irréversibilité partielle ou totale.

\section{Activité physique}

Chute de l'activité physique, notamment pendant les loisirs, et sédentarité croissante sont des composants majeurs et indépendants de la constitution de l'obésité. Leur importance a été sous-estimée. II est intéressant de voir par exemple que pour leurs déplacements quotidiens, près de $50 \%$ des Néerlandais se déplacent à pied ou en vélo contre $10 \%$ des Américains, ce qui pourrait expliquer (en partie) l'énorme différence de prévalence (15\% contre plus de $30 \%$ ) de l'obésité entre les deux pays. Géographie urbaine, TV, jeux vidéo... A cela s'ajoutent sans doute les quantités d'énergie dissipées lors de la gestuelle, sans doute caractéristique de la personnalité de chacun et dont on a pu montrer qu'elles permettent de prédire les capacités d'engraissement. de n'être pas quantitativement sousalimentée :

- la densité énergétique, qui ralentit la satiété et est liée tout autant à la richesse en lipides qu'à la pauvreté en eau ;

- la texture, solide versus liquide, celle-ci ayant la réputation d'être moins satiétogène (ce qui, à calories égales, est loin d'être certain) ;

- la palatabilité, le goût pour les aliments s'ajustant souvent sur le goût des aliments; le plaisir (souvent lié au caractère crémeux et sucré) fait partie heureusement des déterminants importants de l'appétit ;

- la taille des portions qui pousse à finir l'assiette ou la barquette et à vider le verre ;

- I'humeur, les stress, la dépression, facteurs de troubles du comportement alimentaire ;

- sans parler des incitations publicitaires, des savoirs, des idées reçues, des répertoires culinaires liés à la culture et à la symbolique... Le modèle alimentaire français, ou ce qu'il en reste, semble relativement protecteur.

Ainsi I'on peut dire que la prise de poids est souvent la conséquence physiologique «normale » de l'exposition à l'alimentation occidentale dans des conditions de sédentarité « moderne » en l'absence d'une « volonté de fer » pour manger différemment.

Dans les interventions des autres orateurs seront présentés le devenir métabolique des acides gras, le rôle de certains d'entre eux sur le développement du tissu adipeux, les relations entre lipides et appétence, les données épidémiologiques d'intervention, avec l'idée de répondre à la question sous-jacente : peut-on faire des lipides alimentaires un acteur majeur de la constitution de l'obésité ?

\section{Autres facteurs}

Si nutrition et exercice physique occupent le devant de la scène et sont sans aucun doute des acteurs majeurs de l'évolution du poids, d'autres aspects des modifications sociétales récentes doivent sans doute être pris en compte.

Il est remarquable en effet que l'évolution séculaire de la prévalence de l'obésité, qui a pris son essor dans les années 60 (du XXe siècle), montre dans les pays anglo-saxons un parallélisme relatif avec la réduction du temps de sommeil, la prescription d'antidépresseurs, la température moyenne des appartements, la proportion de fongicides dans le lait maternel, l'âge de la mère à la première grossesse, la réduction du tabagisme auxquels on peut ajouter l'élévation du rapport oméga 6/oméga 3. Tous ces éléments ont sans doute un effet spécifique potentiel faible mais ils sont additifs et méritent, tout comme l'hypothèse virale récemment envisagée, davantage de recherche.

En conclusion de ce chapitre, on peut dire que la génétique "propose», l'environnement « impose » plutôt plus que moins et que l'individu ne « dispose » pas forcément des instruments de sa décision personnelle.

\section{Histoire naturelle de l'obésité}

Très schématiquement, on peut décrire plusieurs phases successives (figure 2). Dans un premier temps, l'individu prend du poids sous I'influence du bilan d'énergie positif créé par les facteurs précédemment décrits: c'est la phase dynamique. Au début, l'inflation du tissu adipeux s'opère par l'intermédiaire d'un

\section{Les facteurs alimentaires}

Face à une réduction des dépenses énergétiques, la prise alimentaire dispose de capacités d'ajustement non négligeables; celles-ci ont néanmoins des limites, dans la mesure notamment où le comportement alimentaire n'est pas assujetti qu'aux seules nécessités de I'homéostasie énergétique : de nombreux facteurs conduisent à provoquer un excès d'apports relativement aux dépenses. Tout semble indiquer qu'il n'y a pas d'aliments ou de nutriments qui aient en eux-mêmes une valeur explicative universelle, un bilan d'énergie positif pouvant se constituer de bien des manières. Parmi les facteurs qui contribuent à modifier la prise alimentaire, quantitative ou qualitative, on peut lister les suivants :

- la disponibilité, bien sûr (puisque l'on ne saurait consommer ce qui est inaccessible) qui a fortement augmenté dans nos pays ;

- les prix alimentaires bas, surtout pour les denrées caloriquement denses, permettant à la grande majorité des couches de la population



Figure 2. Histoire naturelle de l'obésité : les différentes phases. 
accroissement de la taille des adipocytes (hypertrophie), puis se met en place une adipogénèse accrue créant des préadipocytes puis de nouveaux adipocytes (hyperplasie) pendant que s'installent diverses modifications neurohormonales et métaboliques: une irréversibilité partielle ou totale est ainsi créée.

Souvent cette phase dynamique est interrompue par des « régimes » qui, lorsqu'ils sont sévères et induisent une perte de poids, $\mathrm{n}^{\prime}$ auront que des effets à court terme vite suivis $d^{\prime} u n e$ reprise pondérale rapide qui aggrave le problème puisque les réserves adipeuses se reconstituent plus vite que la masse maigre, support principal de $80 \%$ des dépenses énergétiques. Pour le patient, sentiment d'échec, culpabilité, découragement, mésestime de soi, dépression parfois, ne facilitent pas un comportement alimentaire adapté. C'est la raison pour laquelle l'objectif thérapeutique devrait viser à la stabilisation du poids plutôt qu'à sa réduction.

Au bout d'un certain temps, au terme d'une évolution linéaire ou plus souvent après plusieurs tentatives de perte de poids (syndrome du yo-yo) le poids finit par se stabiliser, c'est la phase statique, le bilan d'énergie est à nouveau équilibré, les dépenses énergétiques ont augmenté, I'apport alimentaire aussi. Le tissu adipeux à la fois hypertrophique et hyperphasique est progressivement devenu « malade ", générateur d'une insulinorésistance modifiant les équilibres hormonaux, infiltré de cellules inflammatoires sécrétrices de cytokines, etc., tous facteurs essentiels des complications métaboliques de l'obésité. On est ainsi passé d'une inadaptation de certains sujets aux modifications de l'environnement (pas seulement nutritionnel) à une maladie organique du tissu adipeux.

\section{Conclusion}

L'obésité est un état chronique autoentretenu qui relève de mécanismes divers et complexes. Le rôle des facteurs alimentaires est sans doute important mais il est loin d'être le seul en cause dans l'établissement d'un bilan d'énergie positif. Ériger un type d'aliment ou de nutriment lipides ou glucides - en responsable principal et pour toute une population parait bien difficile même si pour certains sujets selon leur terrain génétique et leurs choix alimentaires peuvent être aberrants, l'un ou l'autre peut être en cause.

\section{RÉFÉRENCE}

1. In : Basdevant A, Guy-Grand B, eds. Médecine de l'obésité. Flammarion - Médecine Sciences, 2004 ; (1vol, 431p). 